\section{CLL: Kombinationstherapie langfristig erfolgreich}

Bei körperlich fitten, bisher unbehandelten Patienten mit chronischer lymphatischer Leukämie (CLL) gilt die Chemoimmuntherapie mit Fludarabin, Cyclophosphamid und Rituximab (FCR) als Standard. Nun gibt es Langzeitergebnisse aus der CLL8-Studie.

D ie 2003 begonnene CLL-8-Studie der deutschen CLL-Studiengruppe hatte diesen Standard etabliert. In die Studie waren 817 median 61 Jahre alte CLL-Patienten in körperlich guter Verfassung aufgenommen worden, die noch keine Therapie erhalten hatten. $31 \%$ hatten eine CLL im Binet-Stadium C, 64\% im Stadium B. Bei $63 \%$ der Patienten war das IGHV(,immunoglobulin heavy chain variable“)-Gen nicht mutiert. Primärer Endpunkt war das progressionsfreie Überleben (PFS). Alle Patienten wurden mit 6 Zyklen Fludarabin (F; $25 \mathrm{mg} / \mathrm{m}^{2}$ i.v. an Tag $\left.1-3, \mathrm{q} 4 \mathrm{w}\right)$ plus Cyclophosphamid (C; $250 \mathrm{mg} / \mathrm{m}^{2}$ i.v. an Tag $1-3, q 4 w)$ behandelt. In der Verumgruppe wurde darüber hinaus Rituximab (R;
Zyklus 1: $375 \mathrm{mg} / \mathrm{m}^{2}$ an Tag 0; Zyklus 2-6: $500 \mathrm{mg} / \mathrm{m}^{2}$ an Tag 1) verabreicht.

Nach einer medianen Nachbeobachtungszeit von 5,9 Jahren lag das PFS in der FCR-Gruppe bei 56,8 Monaten und in der FC-Gruppe bei 32,9 Monaten (Hazard Ratio [HR] 0,59; $95 \%$-Konfidenzintervall [95\%-KI] 0,50-0,69; $\mathrm{p}<0,001)$. Bei den Patienten mit Chemoimmuntherapie wurde der Medianwert beim Gesamtüberleben bislang nicht erreicht, in der Vergleichsgruppe lag er bei 86,0 Monaten (HR 0,68, 95\%-KI 0,54-0,89; $\mathrm{p}=0,001$ ).

Bei Patienten mit mutiertem IGHVGen waren sowohl PFS als auch Gesamtüberleben unter der FCR-Kombination im Vergleich zur ausschließlichen Che- motherapie deutlich verbessert (HR $0,47 ; \mathrm{p}<0,001$ bzw. HR 0,62; $\mathrm{p}=0,1)$. Nach 5 Jahren lebten nach der FCR-Therapie noch $66,6 \%$ der Patienten ohne Fortschreiten der Erkrankung, in der Gruppe ohne Rituximab nur 36,2\%. Auf Gesamtansprechrate, Komplettremissionen sowie $\operatorname{MRD}($,minimal residual disease")-Negativität hatte der IGHVGenstatus keinen Einfluss.

Fazit: Körperlich fitte, bisher unbehandelte Patienten mit CLL profitieren auch langfristig mehr von einer Chemoimmuntherapie unter Einschluss des monoklonalen Antikörpers Rituximab als von einer Chemotherapie mit Fludarabin und Cyclophosphamid allein. Das spiegelt sich in der CLL-8-Studie unter anderem in der Überlebensdauer ohne Fortschreiten der Erkrankung wider.

Peter Leiner

Fischer $\mathrm{K}$ et al. Long-term remissions after FCR chemoimmunotherapy in previously untreated patients with CLL: updated results of the CLL8 trial. Blood. 2016;127(2):208-15.

\title{
Hypogammaglobulinämie bei jedem vierten CLL-Patienten
}

\begin{abstract}
Die Hypogammaglobulinämie ist eine bekannte Komplikation bei chronischer lymphatischer Leukämie (CLL), aber ihre Prävalenz zum Zeitpunkt der CLL-Diagnose, ihre Assoziation mit neuen prognostischen Markern sowie der Einfluss auf die Prognose sind bisher nicht systematisch untersucht worden.
\end{abstract}

$\mathrm{n}$ einer retrospektiven Studie wurde der Zusammenhang zwischen Hypogammaglobulinämie und neuen prognostischen Parametern bei 1.485 von Januar 1999 bis Juli 2013 an der MayoClinic behandelten Patienten mit neu diagnostizierter CLL untersucht, bei denen das Serum-Immunglobulin G (IgG) zu Studienbeginn bestimmt worden war.

382 (26\%) der Patienten hatten eine Hypogammaglobulinämie mit einer medianen IgG-Konzentration von $624 \mathrm{mg} /$ dl. Die übrigen 1.103 Patienten (74\%) hatten normale IgG-Spiegel (median $1.040 \mathrm{mg} / \mathrm{dl}$ ). Patienten mit Hypogammaglobulinämie zum Zeitpunkt der Diagnose hatten häufiger ein fortgeschrittenes Rai-Stadium (III-IV; $\mathrm{p}=0,001$ ) und eine höhere Expression von a4-
Integrin, bzw. CD49d ( $\mathrm{p}<0,001)$ als Patienten mit normalen IgG-Spiegeln.

Nach median 4,2 Jahren hatten 32\% der Patienten eine CLL-Therapie erhalten. Die mediane Zeit bis zur ersten Therapie war bei Patienten mit Hypogammaglobulinämie kürzer als bei Patienten mit normalen IgG-Spiegeln (3,8 vs. 7,4 Jahre; $\mathrm{p}<0,001)$. Dennoch unterschied sich das mediane Gesamtüberleben der beiden Gruppen nicht signifikant $(12,8$ vs. 11,3 Jahre; $p=0,73$ ). Die Assoziation zwischen Hypogammaglobulinämie und Überleben blieb unverändert, wenn der Grenzwert für den IgG-Spiegel von $767 \mathrm{mg} / \mathrm{dl}$ auf $500 \mathrm{mg} / \mathrm{dl}$ herabgesetzt wurde. Bei den 1.103 Patienten mit normalen IgG-Spiegeln zum Diagnosezeitpunkt, die keine CLL-Therapie bekamen, lag das Risiko einer erworbenen Hypogammaglobulinämie nach 5 Jahren bei $11 \%$ und nach 10 Jahren bei $23 \%$. Ein nicht mutiertes IGHV(,immunoglobulin heavy chain variable")-Gen und eine hohe CD49d-Expression waren bei diesen Patienten signifikant mit dem Risiko für die Entwicklung einer Hypogammaglobulinämie assoziiert (Hazard Ratio 1,9 bzw. 1,8).

Fazit: Eine Hypogammaglobulinämie liegt bei jedem vierten Patienten mit neu diagnostizierter CLL vor. Zudem entwickelt etwa ein Viertel der CLL-Patienten mit zum Zeitpunkt der Diagnose normalen IgG-Spiegeln im weiteren Verlauf der Erkrankung eine Hypogammaglobulinämie. Deren Vorliegen scheint das Gesamtüberleben jedoch nicht $\mathrm{zu}$ beeinflussen.

Judith Neumaier

Parikh SA et al. Hypogammaglobulinemia in newly diagnosed chronic lymphocytic leukemia: Natural history, clinical correlates, and outcomes. Cancer. 2015;121(17):2883-91. 